\title{
Escala de Identificação com Grupos Alternativos: construção e comprovação da estrutura fatorial
}

\author{
Carlos Eduardo Pimentel \\ Valdiney V. Gouveia \\ Patricia Nunes da Fonseca
}

\begin{abstract}
Resumo
O objetivo deste estudo foi construir e validar uma escala para medir a identificação dos jovens com grupos alternativos. Participaram da pesquisa 548 estudantes do ensino médio, majoritariamente com idade variando de 13 e $19 \operatorname{anos}(97,6 \% ; M=16,2, D P=1,74)$. Estes responderam um bloco de questionários, entre os quais a Escala de Identificação com Grupos Alternativos, que se compõe de sete itens-grupos (bippies, punks, skinheads, headbangers, skatistas, surfistas e funkeiros), respondidos em escala de cinco pontos. Os resultados indicaram uma estrutura unifatorial, com todos os itens apresentando saturações acima de 0,65; este fator geral obteve um eigenvalue de 4,02, explicando 56\% da variância total, com consistência interna ( $\alpha$ de Cronbach) de 0,87 . Portanto, conclui-se que esta é uma medida adequada para fins de pesquisa, sendo útil quando o propósito for conhecer a identificação dos jovens com grupos alternativos, a exemplo dos surfistas e punks.

Palavras-Chave: Grupos alternativos; Identificação grupal; Jovens.
\end{abstract}

\section{Identification with Alternative Groups Scale: Development and validity}

\begin{abstract}
This study aimed at building and validating a scale for measure the youngsters' identification with alternative groups. Participated 548 high-school students, most of them with a mean age of 16 years old $(97.6 \% ; M=16.2$, $S D=1.74)$. They answered a set of questionnaires, among they the Identification with Alternative Groups Scale, composed by seven alternative groups (hippies, punks, skinheads, headbangers, skaters, surfers, and funkeiros), rating in a 5 points scale. Result indicates a one-factor structure, with all items showing factor loadings above of .65. This general factor showed an eigenvalue of 4.02 , accounting to $56 \%$ to the total variance, and obtaining a reliability (Cronbach's $\alpha$ ) of .87 . It was concluded that this an appropriateness measure to research purpose, being useful to knowing the youngsters' identification with alternative groups (e.g., surfers, punks).

Keywords: Alternative groups; Group identification; Youngters.
\end{abstract}

\section{Introdução}

A Identificação Grupal pode ser entendida sinteticamente como "a importância subjetiva do grupo para o indivíduo" (Kiesner, Cadinu, Poulin \& Bucci, 2002, p. 197). Krech, Crutchfield e Ballachey (1975) definem a identificação como "o processo de procurar ser como outro indivíduo ou grupo”, indicando que, pela identificação, o indivíduo passa a incorporar as características de outro indivíduo ou grupo e a manifestar um comportamento semelhante. Estes grupos são chamados de grupos de referência, pois servem de modelo, sendo significativos para o indivíduo; ao não fazer parte deles, o indivíduo que os considera como referência expressa o desejo de se integrar, mostrando atitudes na mesma direção dos grupos (Kiesler \& Kiesler, 1973).

A identificação grupal está na base de vários processos e variáveis psicossociais. Por exemplo, é preponderante no processo de formação, mudança e manutenção das atitudes (Asch, 1977; Freedman, Carlsmith \& Sears, 1970; Lambert \& Lambert, 1972; Siegel \& Siegel, 1957), na auto-estima (Bat-Chava, 1994), nos valores sociais (Gouveia, Albuquerque, Clemente \& Espinosa, 2002), na influência social (Rodrigues, Assmar \& Jablonsky, 2000), nos traços de resposta interpessoal, como a dominância, o exibicionismo, a sociabilidade, bem como na formação de grupos e na liderança (Krech e cols., 1975), na busca por aceitação e evitação da rejeição, na auto-avaliação (Kiesler \& Kiesler, 1973) e nos comportamentos anti-sociais (Benda \& Corwyn, 2000; Hawkins, Catalano \& Miller, 1992; Petraitis, Flay \& Miller, 1995).

Embora muito se saiba sobre a identificação grupal em geral, poucos estudos têm sido realmente

\footnotetext{
${ }^{1}$ Endereço para correspondência:

R. Eduardo Medeiros, 125 - Castelo Branco I - 58050-080 - João Pessoa-PB

E-mail: cedups@bol.com.br
} 
realizados para avaliar a identificação com grupos alternativos específicos (Sussman, Dent \& McCullar, 2000), como aqueles que se vinculam aos estilos musicais alternativos ou não-convencionais (por exemplo, punks, headbangers, hippies). Estes, entretanto, podem ser considerados como formadores de uma cultura de grupo, sistema de significações que incidem, sobretudo, mas não tão-somente, no comportamento de jovens $\mathrm{e}$ adolescentes (ver Pais, 1998). Nas pesquisas em que tais grupos têm sido considerados, observa-se que os jovens que se identificam com eles tendem a apresentar comportamentos anti-sociais, incluindo o uso de álcool e drogas (Costa, 1993; López \& García, 1997; Salem, 1996; Sussman e colaboradores, 2000; Wood, 1999). Sobre esta temática, demanda-se tratar mais detalhadamente.

\section{Identificação grupal e comportamentos anti-sociais}

No conjunto de variáveis pertinentes à explicação dos comportamentos anti-sociais, a influência dos modelos sociais, definidos como pessoas importantes para o jovem, nomeadamente os amigos, a associação com pares desviantes e a família têm se destacado marcantemente (Allison \& cols., 1999; Dishion, Patterson, Stoolmiller \& Skinner, 1991; Fisher \& Fagot, 1993; Heaven, Caputi, Trivellion-Scott \& Swinton, 2000; Miller, 1997; Scaramella, Conger, Spoth \& Simons, 2002; Vitaro, Brendgen \& Tremblay, 2000; Vuchinich, Bank, \& Patterson, 1992; Whright \& Cullen, 2001).

De notória relevância nesses estudos encontrase o Modelo do Desenvolvimento Social, que visa à explicação do uso de drogas e álcool, mas também à explicação da delinqüência e violência juvenis (ver Benda \& Corwin, 2000; Herrenkohl \& cols., 2000; Saner \& Ellickson, 1996). Este quadro explicativo incorpora o pressuposto segundo o qual o tipo da relação com os grupos de referência, aqueles que servem à modelagem do comportamento social, constituem-se em fatores de risco e proteção para estas condutas. Vários resultados de pesquisas acerca do tema apóiam a hipótese do compromisso social ou da sociedade convencional. Segundo essas pesquisas, o compromisso com grupos normativos (por exemplo, familiares, pais, professores), os quais assumem a função de transmissão dos valores sociais ao mesmo tempo em que garantem a harmonia social, é considerado como um fator de proteção para condutas anti-sociais e delitivas (Benda \& Corwyn, 2000; Hawkins, Catalano \& Miller, 1992; Petraitis, Flay \& Miller, 1995; Sussman e cols., 2000). Este compromisso tem início com o processo de identificação grupal.

Recentemente, em pesquisa empírica, Sussman e cols. (2000) verificaram que os considerados "grupos de alto risco", no caso membros de gang, "drogados", pixadores, rappers e metaleiros (roqueiros ou headbangers), obtiveram médias mais altas nas escalas que avaliam uso de álcool e maconha quando comparados com outros grupos juvenis. López e García (1997) encontraram "uma marcada tendência nos sujeitos que consomem drogas ilegais, e fazem uso intenso de álcool, a simpatizar ou pertencer a grupos ou tribos urbanas" (p. 170). É importante destacar que muitos desses grupos são rígidos com relação às normas grupais.

Como tem apontado Arnett (1991, apud McNamara e Ballard, 1999), o grupo de adolescentes fãs de heavy metal, considerando estadunidenses (beadbangers ou batedores de cabeça), é tido como um dos grupos mais "extremos". Grupo "extremo" aqui tem a conotação de um grupo mais radical ou fanático, quando comparado com fãs de outras ramificações do rock. Costa (1993) e Salem (1996), no contexto brasileiro, e Wood (1999), no contexto norte-americano, com base em diversas entrevistas com membros do grupo dos carecas, grupo de neonazistas brasileiros influenciados pelos skinheads ingleses, caracterizam-nos como violentos, autoritários e antidrogas.

Conhecer a influência que exercem estes grupos alternativos (tribos juvenis/urbanas ou subculturas juvenis) propicia um melhor entendimento de diversos comportamentos, crenças, atitudes, valores e normas reforçados e mantidos por seus membros, que agem como uma forte fonte de influência social para adolescentes por fazerem parte ou mesmo por apenas se identificarem com tais grupos. Esse tema pode interessar tanto à psicologia social como à psicologia da adolescência, família, clínica, entre outras. Dessa forma, pretendeu-se construir e validar uma escala para mensurar a identificação diante dos grupos alternativos.

\section{Método}

\section{Participantes}

Tratou-se de uma amostra acidental de 548 estudantes do ensino médio provenientes de escolas públicas $(46,4 \%)$ e privadas $(53,6 \%)$ da cidade de João Pessoa, PB. Destes, $36,7 \%$ cursavam o primeiro ano, $28,8 \%$, o segundo e $34,5 \%$, o terceiro. A maioria era do sexo feminino $(54,9 \%)$ e solteira $(93,6 \%)$, com idades predominantemente entre os 13 e 19 anos $(97,6 \% ; M=16,2, D P=1,74)$.

\section{Instrumento}

Os participantes responderam a uma bateria de instrumentos, dentre os quais a Escala de Identificação com Grupos Alternativos - EIGA. Considerando os propósitos deste artigo, unicamente se fará referência a esta medida. Para sua elaboração foram consideradas duas etapas principais (Pasquali, 2003):

1. Análise de conteúdo. Esta análise, também identificada como teórica, considerou a participação de três juízes, os 
quais deveriam averiguar a pertinência dos grupos listados como representantes dos coletivos jovens da cultura paraibana. Para ser considerado como um juiz, a pessoa deveria participar de algum grupo alternativo ou se relacionar com pessoas destes grupos por, ao menos, cinco anos. A retenção dos itens na escala era considerada com um mínimo de $80 \%$ de acordo entre os juízes. Este procedimento permitiu identificar sete grupos: hippies, punks, skinheads, headbangers, skatistas, surfistas e funkeiros.

2. Análise semântica. Esta contou com a participação de dez estudantes com escolaridade correspondente à sétima série do ensino fundamental. Sua participação foi voluntária e em ambiente específico cedido pela direção da escola, contando com a colaboração dos professores, que os liberaram de suas atividades de sala de aula. A tarefa desses jovens consistia em ler e responder aos itens, avaliando em que medida as instruções eram adequadas e o formato da escala de resposta, com cinco pontos $(0$ = Nada identificado a $4=$ Totalmente identificado).

Estas análises confirmaram a adequação da amostra de grupos alternativos, das instruções de como responder e do formato da escala de resposta. A versão final da escala é apresentada em anexo, a mesma respondida pelos participantes.

\section{Procedimento}

Para a realização da coleta de dados, contataram-se inicialmente as direções das escolas a fim de obter permissão para a aplicação dos questionários. Após seu consentimento, a aplicação foi efetuada por quatro bolsistas de Iniciação Científica (IC) da graduação em Psicologia - UFPB, que receberam um treinamento específico no que se refere ao procedimento da aplicação. Considerando a disponibilidade da escola, as salas de aula foram escolhidas; nelas, os aplicadores solicitaram a colaboração dos estudantes para que respondessem individualmente aos instrumentos. Indicou-se que se tratava de participação voluntária, sendo assegurado a todos o anonimato. As instruções gerais foram expressas oralmente pelos aplicadores, que permaneceram em sala de aula durante toda a coleta, disponibilizando-se a esclarecer eventuais dúvidas quanto à forma de responder. Finalmente, depois de preenchidos os questionários foram dirigidos os agradecimentos de praxe pela colaboração da turma. Em média, 30 minutos foram suficientes para concluir sua participação.

\section{Análises estatísticas}

Tanto a tabulação dos dados como as subseqüentes análises estatísticas foram realizadas por meio do SPSSWIN 12 (Statistical Package for the Social Science).
Foram efetuadas estatísticas descritivas (média, tendência central, dispersão) e análises dos componentes principais (PC). Pelo AMOS 4 foi realizada uma análise fatorial confirmatória, considerando a matriz de correlações e adotando o estimador ML (Maximum Likelihood). Esta estatística oferece alguns indicadores de ajuste do modelo teórico aos dados empíricos (ver Byrne, 1989; Tabachnick \& Fidell, 1996), sendo aqui considerados os seguintes: (1) o $\chi^{2}$ (qui-quadrado) para testar a probabilidade de o modelo teórico se ajustar aos dados, e quanto maior este valor pior o ajustamento; (2) a razão $\chi^{2} /$ g.l. (graus de liberdade) que deve ser preferentemente inferior a 2 para um ajustamento adequado, embora seja admitido um valor de até 5; (3) o Goodness-of-Fit Index (GFI); e (4) o Adjusted Goodness-of-Fit Index (AGFI). Estes últimos variam de 0 a 1 , com valores próximos a 0,90 indicando um ajustamento satisfatório. Análogo ao $\mathrm{R}^{2}$ em regressão múltipla, o GFI indica a proporção de variânciacovariância nos dados explicada pelo modelo.

\section{Resultados}

\section{Análises preliminares}

Para conhecer um pouco sobre a identificação dos participantes com os grupos alternativos, considerando apenas as pontuações que indicam alta identificação, isto é, pontuações 3 e 4, calculou-se o percentual de identificação por grupo. Observou-se que os jovens apresentaram a seguinte magnitude de identificação com os diversos grupos (pessoas) considerados: surfistas $(25,3 \%)$, skatistas (24\%), hippies $(18,1 \%)$, beadbangers (14,5\%), punks (12\%), funkeiros (10,1\%) e, por último, skinheads (4,3\%).

\section{Escala de Identificação com Grupos Alternativos}

Previamente, com o fim de checar a fatorabilidade da matriz de correlações entre os itens desta medida, procedeu-se ao cálculo do KMO (KaiserMeyer-Olkin) e Teste de Esfericidade de Bartlett (ver Tabachnick \& Fidell, 1996). O primeiro trabalha com as correlações parciais das variáveis, devendo ser aceitos índices iguais ou superiores a 0,60 ; o segundo, por outro lado, comprova a hipótese de que a matriz de covariâncias é uma matriz de identidade. Os resultados observados apóiam a realização da subseqüente análise, pois o KMO foi de 0,85 e o Teste de Esfericidade de Bartlett permitiu rejeitar a hipótese desta matriz ser de identidade $\chi^{2}(21)=1646,75 p<0,001$. Neste sentido, procedeu-se à análise de componentes principais (PC), sem fixar o número de fatores a serem extraídos. Os resultados a respeito são apresentados na Tabela 1. 
Tabela 1 - Estrutura fatorial da Escala de Identificação com Grupos Alternativos

\begin{tabular}{lcc}
\hline Grupos de Identificação & Fator & $\mathrm{h}^{2}$ \\
\hline Punk & $\mathrm{I}$ & 0,69 \\
Skatistas & $0,83^{*}$ & 0,66 \\
Metaleiros & $0,81^{*}$ & 0,60 \\
Hippies & $0,78^{*}$ & 0,57 \\
Surfistas & $0,75^{*}$ & 0,53 \\
Skinheads & $0,73^{*}$ & 0,46 \\
Funkeiros & $0,68^{*}$ & 0,43 \\
\hline Número de itens & $0,65^{*}$ & \\
Eigenvalue & 7 & \\
\% Variância & 4,02 & \\
Alfa de Cronbach & 56,40 & \\
\hline
\end{tabular}

Notas: ${ }^{*}$ carga fatorial considerada satisfatória $|0,40| \cdot \mathrm{h}^{2}=$ comunalidade.

Conforme era esperado, a análise PC resultou em um único fator, com eigenvalue igual a 4,02, explicando $56 \%$ da variância total. Todos os itens saturaram satisfatoriamente, isto é, superior a $|0,40|$. De fato, são pesos fatoriais elevados: todos acima de 0,65 . Claramente, refletem uma identificação com grupos alternativos, portanto, assim foi denominado este fator. Sua consistência interna (precisão) é também satisfatória (alfa de Cronbach, $\propto=0,87$ ).

Corroborando a estrutura unidimensional da EIGA

Finalmente, embora existam evidências suficientes da adequação da Escala de Identificação com Grupos
Alternativos, sempre é possível se especular acerca dos procedimentos estatísticos exploratórios aqui empregados. Além disso, uma análise PC é favorável ao suposto de unidimensionalidade, maximizando as comunalidades que vão resultar em um grande fator/componente. Portanto, para dirimir possíveis dúvidas, decidiu-se igualmente realizar uma Análise Fatorial Confirmatória (AFC). Neste caso, os dados de entrada compreenderam as correlações entre os sete grupos alternativos, tendo sido adotado o estimador ML. Considerando a amostra total $(n=548)$, através da rotina Sample do SPSSWIN, foi selecionada uma amostra aleatória de 200 participantes, quantidade que atende às exigências deste tipo de análise (Watkins, 1989).

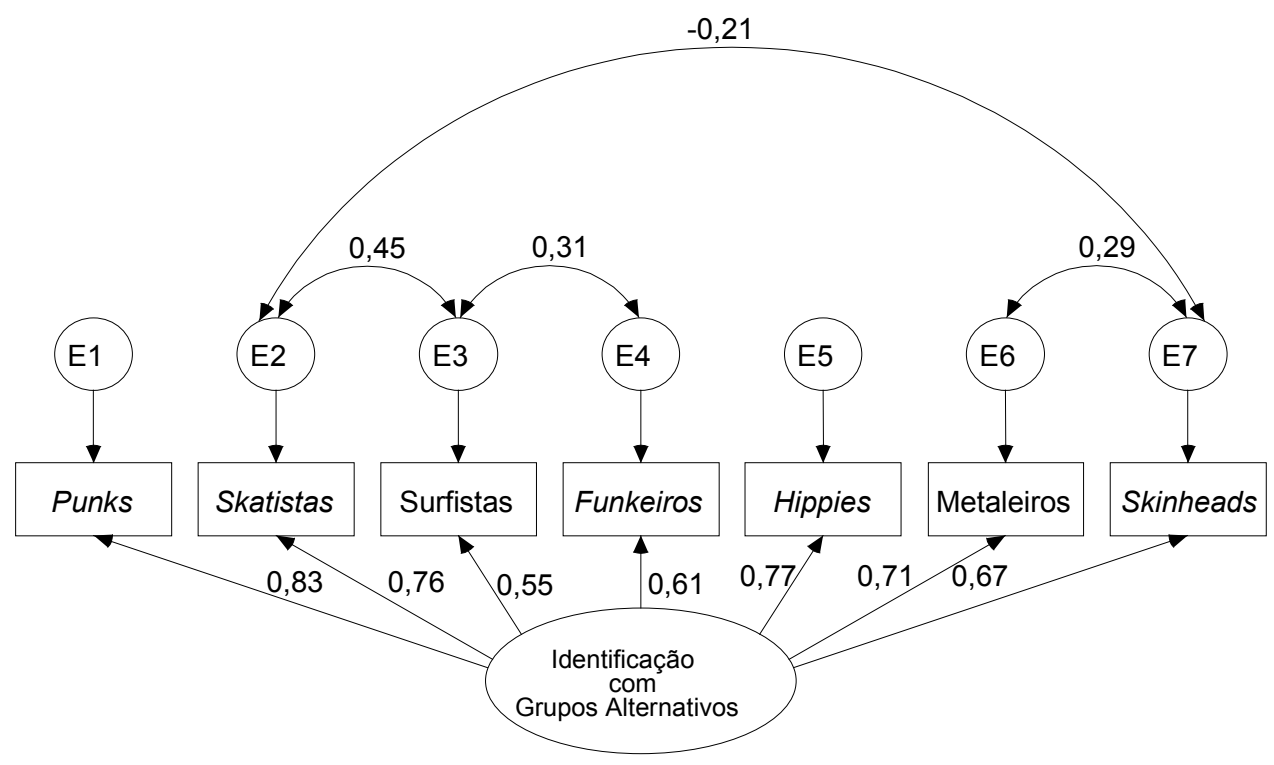

Figura 1 - Comprovação da unidimensionalidade da EIGA 
Como é possível observar nessa figura, os pesos fatoriais (lambdas) são todos positivos, sendo estatisticamente diferentes de zero $(t>1,96, p<0,05)$. Os indicadores de bondade de ajuste permitem apoiar a concepção de uma estrutura unidimensional para a Escala de Identificação com Grupos Alternativos: $\chi^{2}(10)=15,25$, $p=0,12 ; \chi^{2} / \mathrm{g} . \mathrm{l} .=1,52 ; G F I=0,98$ e $A G F I=0,94$.

\section{Discussão}

Nenhum instrumento foi encontrado em periódicos nacionais e internacionais para mensurar a identificação com grupos alternativos, também denominados como anticonvencionais. Neste sentido, planejou-se a realização do presente estudo, cujo objetivo principal foi elaborar uma medida para avaliar a identificação que os jovens apresentam com estes grupos (por exemplo, skinheads, headbangers). Diante dos resultados, pensa-se que o mesmo tenha sido cumprido. Não obstante, cabe apontar eventuais limitações desta pesquisa, a exemplo do número reduzido de itens empregados nessa escala, embora tenham sido contemplados grupos de certa saliência na cena social; figuras clássicas como os hippies ou punks ainda seguem sendo influentes nos tempos hodiernos, mormente no mundo adolescente. Por outro lado, seus estilos, paradoxalmente às suas "filosofias de vida", são incorporados pelo mundo da moda, e então engolidos pela tão criticada indústria cultural. É igualmente importante assinalar que não se contou com uma fundamentação teórica axiomatizada para esta medida, dado que, como se aludiu previamente, a literatura sobre grupos alternativos é escassa na psicologia. Neste sentido, admite-se aqui um procedimento essencialmente empirista. Este aspecto, entretanto, talvez não tenha sido problemático para o conceito de identificação, pois sua definição científica é muito próxima à corriqueira (Kiesner \& cols., 2002), o que facilitou a utilização do conceito e a compreensão das instruções da escala.

Outro dado importante acerca desta medida foi que o item que representa o grupo skinheads (carecas) suscitou alguns questionamentos durante o processo de coleta de dados. Embora não tenha sido contabilizado, um número considerável dos participantes do estudo perguntou quem seriam estes "personagens". Porém este aspecto pode ser relativizado, pois certamente se deve ao contexto cultural em que a presente pesquisa se desenvolveu. Provavelmente este item não apresentaria problemas em amostras do Sul ou Sudeste do país, onde os carecas são figuras mais presentes, muitas vezes envolvidos em atos de vandalismo (Costa, 1993; Salem, 1996). Neste sentido, espera-se que, se a presente pesquisa for reaplicada em São Paulo, por exemplo, a denominação skinheads seja identificada e compreendida mais facilmente.
Apesar do antes comentado, esta primeira versão da Escala de Identificação com Grupos Alternativos pode ser adequadamente empregada com fins de pesquisa. As saturações dos itens no fator único (comunalidade) assim o sugerem. Esta estrutura foi responsável por mais de $50 \%$ de variância explicada do construto correspondente, e apresentou um índice de confiabilidade aceitável. Os grupos listados parecem representar adequadamente a imagem estereotipada dos adolescentes, que buscam "ser diferentes" (Coelho Júnior, 2001), sendo uma das formas de sê-lo participar de atividades "emocionantes", que liberam a adrenalina, como esportes radicais (por exemplo, o skate) ou grupos tidos como desviantes, antiautoritários, ou que mostrem repúdio à família, aos "valores morais", à ordem estabelecida, ao sistema dominante, etc. (por exemplo, os punks ou hippies).

A identificação com esses grupos pode ser entendida como um fator de vulnerabilidade (ou de risco) para comportamentos socialmente desviantes, porém podem ser ainda acrescentados outros grupos a esta escala. Por exemplo, os clubers, que, semelhantemente aos demais grupos, podem também ser caracterizados com relação às músicas que preferem, entre outros elementos, como a roupa, o tipo de cabelo, a linguagem - enfim, um estilo próprio que diz respeito ao mantimento de atitudes e crenças normativas peculiares que conferem identidade ao grupo (Friedlander, 2002; Pais, 1998; Pimentel, Gouveia \& Vasconcelos, no prelo; Souza, 1995). Eles participam, juntamente com indivíduos que conformam outros grupos, de festas noturnas em boates ao ritmo da música techno, das chamadas festas rave (do inglês delírio). Em tais festas são bastante comuns - sobretudo em grandes cidades, mas não tãosomente - o abuso de álcool e a utilização de drogas sintéticas, como o ecstasy (ver Artoni, 2003).

De fato, é desejável que se realizem outras pesquisas que considerem amostras maiores e mais diversificadas, incluindo pessoas de diferentes idades, escolaridades ou mesmo religiões. Seria igualmente interessante obter dados de culturas. Neste caso, poderse-ia, por exemplo, comparar culturas em que se verifica um maior número de grupos juvenis com aquelas mais convencionais, ou mais restritivas com relação a esses grupos, ou mesmo contrastar culturas mais individualistas com aquelas de corte mais coletivista, como é o caso do Brasil e/ou Espanha (Gouveia, Albuquerque, Clemente \& Espinosa, 2002). Podem-se destacar ainda novas direções para estudos que visem conhecer mais acerca da identificação com grupos alternativos, associando-a com traços de personalidade, por exemplo, busca de sensações ou extroversão (McNamara \& Ballard, 1999; Vasconcelos, 2004) ou valores, por exemplo, os de experimentação e normativos (Formiga, 2002; Romero, 
Sobral, Luengo \& Marzoa, 2001). Também poderia ser útil conhecer as atitudes dos jovens identificados com os grupos alternativos ante a política, o sistema capitalista, a polícia e a lei, ou mesmo a criminalidade. Finalmente, no âmbito da medição, poderia ser adequado desenvolver uma medida com estímulos iconográficos dos grupos alternativos, isto é, representando-os por meio de imagens típicas em lugar de simplesmente denominá-los com um rótulo.

\section{Agradecimentos}

O presente artigo contou com o apoio da CAPES e do CNPq mediante bolsas de Mestrado e Produtividade em Pesquisa, concedidas à Carlos Eduardo Pimentel e Valdiney V. Gouveia, respectivamente.

\section{Referências}

Allison, K. W., Crawford, I., Leone, P. E., Trickett, Perez-Febles, A., Burton, L. M. \& Blanc, R. L. (1999). Adolescent substance use: Preliminary examinations of school and neighborhood. American Journal of Community Psychology, 27(2), 111-129.

Artoni, C. (2003). Drogas: precisamos delas? Galileu, 3, 32-37.

Asch, S. E. (1977). Psicologia social. São Paulo, SP: Ed. Nacional (Originalmente publicado em 1952).

Bat-Chava, Y. (1994). Group identification and selfesteem of deaf adults. Personality and Social Psychology Bulletin, 20(5), 494-502.

Benda, B. B. \& Corwyn, R. F. (2000). A test of the validity of delinquency syndrome construct in a homogeneous sample. Journal of Adolescence, 23(4), 497-511.

Byrne, B. M. (1989). A primer of LISREL: Basic applications and programming for confirmatory factor analytic models. New York: Springer-Verlag.

Coelho Júnior, L. L. (2001). Uso potencial de drogas em estudantes do ensino médio: suas correlações com as prioridades axiológicas (Dissertação de Mestrado). João Pessoa, PB: Universidade Federal da Paraíba - Departamento de Psicologia.

Costa, M. R. (1993). Os "carecas do subúrbio": caminhos de um nomadismo moderno. Petrópolis, RJ: Vozes.

Dishion, T. J., Patterson, G. R., Stoolmiller, M. \& Skinner, M. L. (1991). Family, school, and behavioral antecedents to early adolescent involvement with antisocial peers. Developmental Psychology, 27(1), 172-180.

Fisher, P. A. \& Fagot, B. I. (1993). Negative discipline in families: A multidimensional risk model. Journal of Family Psychology, 7(2), 250-254.

Formiga, N. S. (2002). Condutas anti-sociais e delitivas: uma explicação baseada nos valores humanos (Dissertação de Mestrado). João Pessoa, PB: Universidade Federal da Paraíba - Departamento de Psicologia.

Freedman, J. L., Carlsmith, J. M. \& Sears, D. O. (1970). Psicologia social. São Paulo, SP: Cultrix.

Friedlander, P. (2002). Rock and roll: uma história social. Rio de Janeiro, RJ: Record.

Gouveia, V. V., Albuquerque, F. J. B., Clemente, M. \& Espinosa, P. (2002). Human values and social identities. International Journal of Psychology, 37(6), 333-342.

Hawkins, J. D., Catalano, R. F. \& Miller, J. Y. (1992). Risk and protective factors for alcohol and other drug problems in adolescence and early adulthood: Implications for substance abuse prevention. Psychological Bulletin, 112(1), 64-105.

Heaven, P. C. L., Caputi, P., Trivellion-Scott, D. \& Swinton, T. (2000). Personality and group influences on self-reported delinquent behavior. Personality and Individual Differences, 28(6), 1143-1158.

Herrenkohl, T. I., Maguin, E., Hill, K. G., Hawkins, J. D., Abbott, R. D. \& Catalano, R. F. (2000). Developmental risk factors for youth violence. Journal of Adolescent Health, 26(3), 176-186.

Kiesler, C. A. \& Kiesler, S. B. (1973). Conformismo. São Paulo, SP: Edgar Blucher (Originalmente publicado em 1969).

Kiesner, J., Cadinu, M. R., Poulin, F. \& Bucci, M. (2002). Group identification in early adolescence: Its relation with peer adjustment and its moderator effect on peer influence. Cbild Development, 73(1), 196-208.

Krech, D., Crutchfied, R. S. \& Ballachey, E. L. (1975). $O$ indivíduo na sociedade. São Paulo, SP: Pioneira (Originalmente publicado em 1962).

Lambert, W. W. \& Lambert, W. E. (1972). Psicologia social. Rio de Janeiro, RJ: Zahar (Originalmente publicado em 1964).

López, M. J. M. \& García, J. M. M. (1997). Violência juvenil. Em A. M. González, M. M. J. García, J. S. L. López, \& M. M. J. Carrasco (Orgs.). Comportamientos de riesgo: violência, práticas sexuales de riesgo y consumo de drogas ilegales en la juventud (pp. 47-77). Madrid, ESP: Entinema.

McNamara, L. \& Ballard, M. E. (1999). Resting arousal, sensation seeking, and music preference. Genetic, Social, and General Psychology Monographs, 125(3), 229-250. 
Miller, P. (1997). Family structure, personality, drinking, smoking and illicit drug use: a study of UK teenagers. Drug and Alcohol Dependence, 45(14), 121-129.

Pais, J. M. (1998). Culturas juvenis. Lisboa, PO: Imprensa Nacional Casa da Moeda.

Pasquali, L. (2003). Psicometria: teoria dos testes na psicologia e na educação. Petrópolis, RJ: Vozes.

Petraitis, J., Flay, B. R. \& Miller, T. Q. (1995). Reviewing theories of adolescent substance use: Organizing pieces in the puzzle. Psychological Bulletin, 117(1), 67-86.

Pimentel, C. E., Gouveia, V. V. \& Vasconcelos, T. C. (no prelo). Preferência musical, atitudes frente ao uso de maconha e comportamentos anti-sociais entre adolescentes. Estudos de Psicologia/PUCCAMP.

Rodrigues, A., Assmar, M. E. L. \& Jablonsky, B. (2000). Psicologia social. Petrópolis, RJ: Vozes.

Romero, E., Sobral, J., Luengo, M. A. \& Marzoa, J. A. (2001). Values and antisocial behavior among Spanhish adolescents. The Journal of Genetic Psychology, 162(1), 20-40.

Salem, H. (1996). As tribos do mal: o neonazismo no Brasil e no mundo. São Paulo, SP: Atual.

Saner, H. \& Ellickson, P. (1996). Concurrent risk factors for adolescent violence. Journal of Adolescent Health, 19(2), 94-103.

Scaramella, L. V., Conger, R. D., Spoth, R. \& Simons, R. L. (2002). Evaluation of a social contextual model of delinquency: A cross-study replication. Child Development, 73(1), 175-195.

Siegel, A. E. \& Siegel, S. (1957). Reference group, membership group, and attitude change. Em N. Warren \& M. Jahoda (Eds.). Attitudes (pp. 129-138).
England: Penguin Modern Psychology Readings.

Souza, A. M. A. (1995). Cultura rock e arte de massa. Rio de Janeiro, RJ: Diadorim.

Sussman, S., Dent, C. W. \& McCullar, W. J. (2000). Group self-identification as a prospective predictor of drug use and violence in high-risk youth. Journal of Addictive Behaviors, 14(2), 192-196.

Tabachnick, B. G. \& Fidell, L. S. (1996). Using multivariate statistics. California, EUA: Allyn \& Bacon.

Vasconcelos, T. C. (2004). Traços de personalidade e valores bumanos como explicadores das condutas antissociais $e$ delitivas (Dissertação de Mestrado). João Pessoa, Pb: Universidade Federal da Paraíba.

Vitaro, F., Brendgen, M. \& Tremblay, R. E. (2000). Influence of deviant friends on delinquency: Searching for moderator variables. Journal of Abnormal Child Psychology, 28(4), 313-325.

Vuchinich, S., Bank, L. \& Patterson, G. R. (1992). Parenting, peers, and the stability of antisocial behavior in preadolescent boys. Developmental Psychology, 28(3), 510-521.

Watkins, D. (1989). The role of confirmatory factor analysis in cross-cultural research. International Journal of Psychology, 24(4), 685-701.

Whright, J. P. \& Cullen, F. T. (2001). Parental efficacy and delinquent behavior: Do control and support matter? Criminology, 39(3), 677-705.

Wood, R. T. (1999). The indigenous, nonracist origins of the American skinhead subculture. Youth \& Society, 31(2), 131-151.

Recebido em novembro de 2004 Aprovado em outubro de 2005

Sobre os autores:

Carlos Eduardo Pimentel concluiu o mestrado em Psicologia Social pela Universidade Federal da Paraíba em 2004. Seus interesses de pesquisa incluem preferência musical, culturas juvenis, atitudes e comportamentos antisociais.

Valdiney V. Gouveia doutorou-se em Psicologia Social pela Universidade Complutense de Madrid em 1998. É professor do Departamento de Psicologia da Universidade Federal da Paraíba. Seus interesses de pesquisa incluem os valores humanos, individualismo-coletivismo, traços de personalidade e comportamentos anti-sociais.

Patrícia Nunes da Fonseca é doutoranda em Psicologia Social pela Universidade Federal da Paraíba. Seus interesses de pesquisa incluem valores humanos, desempenho acadêmico, ajustamento escolar e bem-estar subjetivo. 


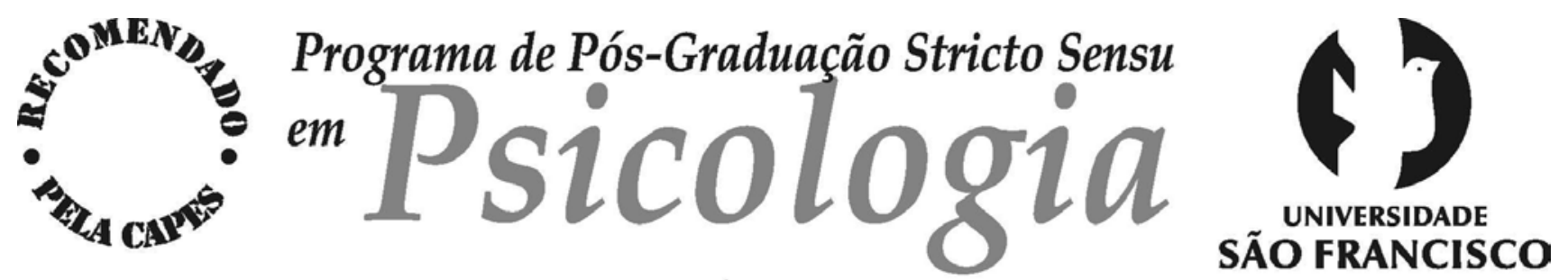

UAACH - Unidade Acadêmica da Área de Ciências Humanas e Sociais Câmpus de Itatiba

\section{Objetivos}

O Programa de Mestrado e Doutorado em Psicologia tem como objetivo (a) formar pesquisadores em nível de Mestrado e Doutorado para que estes possam atuar em serviços e instituições públicas e particulares, a fim de desenvolver atividades de pesquisa, consultorias, assessorias e prestação de serviços que envolvam avaliação psicológica e psicoeducacional; (b) proporcionar experiências que contribuam para a formação de docentes do ensino superior com conhecimento especializado na área de avaliação e medidas; (c) propiciar experiências e discussões teóricas envolvendo construção, aprimoramento e utilização de instrumentos e procedimentos de avaliação psicológica e psicoeducacional em situações específicas e (d) prestar serviços, assessorias e consultorias visando à inserção da universidade na comunidade e contribuindo para o desenvolvimento de serviços de excelência em avaliação psicológica e psicoeducacional na região.

\section{DESTINADO A}

Profissionais graduados em Psicologia e áreas correlatas.

\section{COMISSÃO DE PÓS-GRADUAÇÃO EM PSICOLOGIA (CPG)}

Prof. Dr. Makilim Nunes Baptista (Presidente)

Profa. Dra. Ana Paula Porto Noronha

Prof. Dra. Alessandra Gotuzzo Seabra Capovilla

Profa. Dra. Maria Cristina Rodrigues Azevedo Joly (Suplente)

\section{LINHAS DE PESQUISA}

1. Construção, Validação e Padronização de Instrumentos de Medida Corpo Docente: Profa. Dra. Ana Paula Porto Noronha

Profa. Dra. Claudette Maria Medeiros Vendramini

Prof. Dr. Fermino Fernandes Sisto

Prof. Dr. Ricardo Primi

\section{Avaliação Psicológica em Contextos da Saúde Mental}

Corpo Docente: Profa. Dra. Alessandra Gotuzo Seabra Capovilla

Profa. Dra. Anna Elisa de Villemor Amaral

Prof. Dr. Claúdio Garcia Capitão

Prof. Dr. Makilim Nunes Baptista

\section{Avaliação em Psicologia Educacional}

Corpo Docente: Profa. Dra. Acácia Aparecida Angeli dos Santos

Prof. Dr. Fermino Fernandes Sisto

Profa. Dra. Maria Cristina Rodrigues Azevedo Joly

Prof. Dr. Ricardo Primi 